\title{
Quantification of CatSper I expression in human spermatozoa and relation to functional parameters
}

\author{
L. Tamburrino', S. Marchiani ', E. Vicini' ${ }^{2}$, B. Muciaccia'2 ${ }^{2}$ M. Cambi ${ }^{\text {, }}$ \\ S. Pellegrini' ${ }^{3}$, G. Forti ${ }^{1}$, M. Muratori ', and E. Baldi I,* \\ 'Department of Biomedical Experimental and Clinical Sciences, Center of Excellence DENOthe, University of Florence, Viale Pieraccini 6 , \\ Florence I-50 139, Italy ${ }^{2}$ Fondazione Pasteur Cenci Bolognetti, Department of Anatomy, Histology, Forensic Medicine and Orthopedic, \\ Section of Histology, Sapienza University of Rome, Italy ${ }^{3}$ Centro Procreazione Assistita 'Demetra' of Florence, Via Caccini I8, \\ Florence I-50139, Italy \\ *Correspondence address. E-mail: elisabetta.baldi@unifi.it
}

Submitted on December 17, 2014; resubmitted on April 2, 2015; accepted on April 20, 2015

STUDY QUESTION: Is CatSperl expression in human spermatozoa related to semen parameter values and sperm functions? SUMMARY ANSWER: CatSperl expression is positively related to progressive and hyperactivated $(\mathrm{HA})$ motility, $\left[\mathrm{Ca}^{2+}\right]_{i}$ responsiveness to progesterone but not the acrosome reaction (AR).

WHAT IS KNOWN ALREADY: The role of cationic channel of sperm (CatSper) in sperm functions is clear in animal models but less defined in human sperm cells. Current knowledge is mostly based on low specificity CatSper inhibitors showing agonistic and toxic effects on human spermatozoa and is thus of little help in clarifying the role of the CatSper channel in human sperm functions.

STUDY DESIGN, SIZE, DURATION: CatSperl protein expression was evaluated in I I 5 men undergoing semen analysis for couple infertility. CatSperl expression was related to routine semen parameters, motility kinematic parameters and basal and progesterone-stimulated $\left[\mathrm{Ca}^{2+}\right]_{\mathrm{i}}$ and the AR.

PARTICIPANTS/MATERIALS, SETTING, METHODS: CatSperl expression was evaluated ( $n=85$ normozoospermic, $n=30$ asthenozoospermic patients) by immunofluorescence coupled to flow cytometry leading to quantitative measurement of the percentage of ejaculated sperm cells expressing the protein. Semen analysis was evaluated according to World Health Organization guidelines. Kinematic parameters were evaluated by a computer-aided sperm analysis system. $\left[\mathrm{Ca}^{2+}\right]_{i}$ was measured by a spectrofluorimetric method in fura-2-loaded spermatozoa. The AR was evaluated in live sperm cells by fluorescent-labeled lectin.

MAIN RESULTS AND THE ROLE OF CHANCE: CatSperl protein expression in spermatozoa was reduced in asthenozoospermic men (mean \pm SD: $53.0 \pm 15.5 \%, n=30$ versus $67.9 \pm 17.1 \%$ in normozoospermic, $n=85, P<0.01$ ) and was significantly correlated with progressive $(r=0.36, P<0.001)$, total $(r=0.35, P<0.00 \mathrm{I})$ and HA $(r=0.4 \mathrm{I}, P<0.005)$ motility. In addition to a higher percentage of spermatozoa not expressing CatSper I, asthenozoospermic men showed a large number of spermatozoa with immunofluorescent signal localized outside the principal piece compared with those in normozoospermia. A significant positive correlation was found between CatSper I protein expression and the increase of $\left[\mathrm{Ca}^{2+}\right]_{\mathrm{i}}$ in response to progesterone $(r=0.36, P<0.05, n=40)$ but not with basal $\left[\mathrm{Ca}^{2+}\right]_{\mathrm{i}}$. No correlation was found with the $A R$, either basal or in response to progesterone.

LIMITATIONS, REASONS FOR CAUTION: The study is partly descriptive. Furthermore, we cannot rule out the possibility that some round cells remain after a single round of $40 \%$ density gradient centrifugation or that this step may have removed some defective or slow swimming sperm, and therefore this preparation may not be representative of the entire sperm sample. Although our data suggest that CatSperl may be a useful marker for infertility, and a possible contraceptive target, any clinical application is limited without further research.

WIDER IMPLICATIONS OF THE FINDINGS: Our results demonstrate an association of CatSperl expression with human sperm progressive and $\mathrm{HA}$ motility and provide preliminary evidence that lack of expression or mislocalization of CatSper I in spermatozoa may be involved in the pathogenesis of asthenozoospermia. However, mechanistic studies are needed to confirm that the correlations between CatSperl expression and sperm functions are causative.

(C) The Author 2015. Published by Oxford University Press on behalf of the European Society of Human Reproduction and Embryology. All rights reserved. 
STUDY FUNDING/COMPETING INTEREST(S): Supported by grants from Ministry of University and Scientific Research (PRIN project to E.B. and FIRB project to S.M.) and by Regione Toscana (to G.F.). L.T. was recipient of a grant from Accademia dei Lincei (Rome, Italy). The authors have no conflicts of interest to declare.

Key words: CatSper / asthenozoospermia / sperm motility / hyperactivation

\section{Introduction}

The cationic channel of sperm (CatSper) is a well-characterized calcium channel in mammalian spermatozoa: it is a sperm-specific (Quill et al., 200I), $\mathrm{pH}$-sensitive (Kirichok et al., 2006), voltage-gated calcium channel (Quill et al., 200 I), located in the principal piece of the flagellum (Ren et al., 200 I; Smith et al., 20 I3; Tamburrino et al., 20 I4). In view of the key role of calcium in most sperm functions necessary for successful fertilization, CatSper appears as an interesting target for the development of new therapeutic strategies for treatment of male infertility. In human (but not mouse) sperm cells CatSper is strongly activated by progesterone (Lishko et al., 20 I I; Strunker et al., 20 I I), the female steroid hormone released by cumulus cells surrounding the egg, involved in the fertilization process (Baldi et al., 2009, 20I I). Since men with a reduced sperm-responsiveness to progesterone show reduced natural (Tesarik and Mendoza, 1992; Falsetti et al., 1993) and assisted reproduction (Krausz et al., 1996; Jacob et al., 1998) fertility, an impaired CatSper expression or function leading to a reduced ability to initiate the calcium signaling cascade and the acrosome reaction (AR) process can be hypothesized. However, while substantial progress has been achieved in understanding the physiological role of CatSper in mouse sperm functions, the situation is still confused concerning human spermatozoa.

In the mouse, knockout of any of the CatSper family of genes leads to infertility because of decreased maintenance of motility and lack of achievement of HA motility (Ren et al., 200 I; Qi et al., 2007; Jin et al., 2007). Moreover, although initial studies in CatSper-null mice did not support an involvement of CatSper in the tyrosine phosphorylation increase during sperm capacitation (Carlson et al., 2003; Quill et al., 2003), a recent study (Chung et al., 20I4) analyzing the spatial organization of CatSper in the flagellum in association with signaling activation demonstrated a clear involvement of the channel in such a function. More recently, a role for CatSper in rheotaxis has been also proposed (Miki and Clapham, 2013). Conversely, the involvement of CatSper in the AR of mouse sperm cells has been excluded on the basis of similar percentages of the AR in response to an ionophore in both wild-type and CatSper-null spermatozoa (Xia et al., 2007). In human spermatozoa, the role of CatSper in $\mathrm{HA}$ is still controversial, as in vitro studies where the channel has been stimulated either with progesterone or by raising intracellular $\mathrm{pH}$ did not lead to increased hyperactivation parameters in all samples (Alasmari et al., 20I3a,b; Tamburrino et al., 20I4), and incubation with available CatSper inhibitors led to inconsistent results (SagarePatil et al., 2012, 2013; Tamburrino et al., 2014). Similarly, for progesterone-stimulated ARs, contrasting results have been reported on the effects of CatSper inhibitors (Sagare-Patil et al., 2012, 2013; Tamburrino et al., 20 I4). Inconsistencies in the results with CatSper inhibitors might be due to their poor specificity. Both NNC55-0396 and Mibefradil also block human sperm $\mathrm{K}^{+}$currents (Mansell et al., 20I4) and evoke $\mathrm{Ca}^{2+}$ responses themselves, by rising intracellular $\mathrm{pH}$, therefore acting as partial CatSper agonists (Brenker et al., 20 I 2; Tavares et al.,
20 I3). In addition, we have demonstrated a toxic effect of Mibefradil on human spermatozoa at the concentrations used to inhibit CatSper (Tamburrino et al., 20I4). Conversely, the reason behind reduced or absent responses to CatSper stimuli in some men is unclear. Few men with CatSperl or CatSper2 mutations that lead to a truncated protein have been described in the literature. These men show reduced or absent sperm motility and frequent oligozoospermia, although the two phenotypes are not severe or have not been evaluated or reported for all the subjects with the deletions (Avidan et al., 2003; Avenarius et al., 2009; Hildebrand et al., 2010; Jaiswal et al., 20l4). In one of these patients, showing severe asthenoteratozoospermia due to tail abnormalities and abnormal motility patterns, no basal or progesterone-stimulated $\mathrm{Ca}^{2+}$ currents were detectable (Smith et al., 20I3). We recently demonstrated that a higher percentage of spermatozoa expresses CatSperl after swim-up selection and that a positive correlation exists between CatSperl protein expression, evaluated by western blot analysis, and the percentage of progressive motility, although such a result was obtained in a small number of subjects (Tamburrino et al., 20।4). Interestingly, in non-motile mouse spermatozoa, the CatSper channel is degraded or mislocalized (Chung et al., 20I4). Overall these studies suggest that low, absent or mislocalized CatSper expression in spermatozoa may be involved in determining an asthenozoospermic phenotype and low HA and could explain the lack of response or reduced responses of spermatozoa to CatSper stimuli in some subjects (Alasmari et al., 20।3a,b; Tamburrino et al., 20।4).

To better understand the role of CatSper in human sperm functions, we directly investigated the relationships between the percentage of sperm cells expressing CatSper I protein, as evaluated by immunofluorescence staining coupled to flow cytometry, sperm motility and $\mathrm{HA}$, as well as basal and progesterone-stimulated intracellular calcium levels and AR. In addition, we re-examined CatSper I localization in spermatozoa from normo- and asthenozoospermic men by fluorescence microscopy.

\section{Materials and Methods}

\section{Chemicals}

Human tubal fluid (HTF) medium and human serum albumin (HSA) were purchased from Celbio (Milan, Italy). Bovine serum albumin (BSA) was purchased from ICN Biomedicals (Solon, OH, USA). PureSperm was obtained from Nidacon International (Mölndal. Sweden). Paraformaldehyde (PFA) was obtained from Merck Chemicals (Milan, Italy). Rabbit anti-CatSperI polyclonal antibody was obtained from Santa Cruz Biotechnology (Dallas, TX, USA). Fluorescein isothiocyanate (FITC)-conjugated goat anti-rabbit immunoglobulin (lg) G was obtained from Southern Biotech (Birmingham, AL, USA). Fura 2/AM, TOTO-3 dye and Alexafluor 488 goat anti-rabbit secondary antibody, LI 0120 LIVE/DEAD ${ }^{\circledR}$ Fixable Far Red Dead Cell Stain Kit were obtained from Molecular Probes (Life Technology, Monza, Italy). Anti-human acrosin antibody was obtained from Biosonda Corp. (Santiago, Chile). 
Donkey Cy3-conjugated anti-mouse secondary antibody was obtained from Jackson ImmunoResearch (Suffolk, UK). Propidium iodide (PI) was obtained from Calbiochem (Nottingham, UK). Rabbit serum was obtained from Signet Laboratories (Hayward, CA, USA). Normal goat serum (NGS), normal donkey serum (NDS), progesterone, FITC-labeled Arachis hypogea (peanut) lectin, digitonin, EGTA and Triton were obtained from Sigma Chemical Co. (St. Louis, MO, USA).

\section{Semen collection and preparation}

Semen samples were obtained [according to World Health Organization (WHO) criteria (WHO, 20I0)] from a total of II 5 consecutive patients undergoing routine semen analysis for couple infertility, in the Andrology laboratory of the University of Florence, Italy, after obtaining written informed patient's consent to allow use of the remaining semen after completion of the analysis for research purposes. Semen parameters were assessed by optical microscopy and samples were divided into two groups: normozoospermic $(n=85)$ and asthenozoospermic $(n=30)$, on the basis of progressive $(a+b)$ sperm motility $<32 \%$ and viability $>58 \%$, as the fifth centile of reference values of $\mathrm{WHO}$ (WHO, 20I0). Patients' age and standard semen parameter values for the two groups of men are shown in Table I. The two groups of patients were comparable in age but differed in semen quality. Asthenozoospermic men showed a reduced motility (both progressive and hyperactivated (HA)), a lower sperm concentration and total number per ejaculate and a worse sperm morphology than normozoospermic men. Since all the subjects included in the study were male partners of infertile couples of unknown fertility potential they are indicated as sub-fertile.

In experiments to evaluate motility by computer-aided sperm analysis (CASA), intracellular calcium concentrations $\left(\left[\mathrm{Ca}^{2+}\right]_{i}\right)$ and $A R$, semen samples were selected by a $40 \%$ gradient fraction of PureSperm, prepared in $\mathrm{HTF}$ with $10 \% \mathrm{HSA}$ and centrifuged at $500 \mathrm{~g}$ for 30 min at $26^{\circ} \mathrm{C}$ to eliminate the round cells and debris present in semen that interfere with analysis by CASA (ESHRE, 1998). The resulting 40\% pellet was collected and washed in HTF-10\% HSA medium. Each sample was carefully evaluated under microscopy for effective reduction of germ cells and leukocytes and for maintenance of the initial motility, or otherwise discarded. After selection, the percentage progressive motility was closely correlated with the same parameter at the beginning of sample processing $(r=0.78, P<0.00 \mathrm{I}, n=60)$, although the number of recovered sperm did not allow us to perform $A R$ and $\left[\mathrm{Ca}^{2+}\right]_{\mathrm{i}}$ experiments in all the samples.

\section{Immunofluorescence staining and detection of CatSper I}

The percentage of CatSperl-expressing sperm cells was determined by immunofluorescence staining coupled to flow cytometry in all the 115 samples. A total of $10 \times 10^{6}$ spermatozoa from whole semen were fixed in PFA [500 $\mu$ l, 4\% $\mathrm{w} / \mathrm{v}$ in phosphate-buffered saline (PBS), $\mathrm{pH} 7.4$ ] for $30 \mathrm{~min}$ at room temperature. After being washed twice in I\% ( $/ / \mathrm{v})$ NGSPBS, sperm cells were permeabilized with $0.1 \%(\mathrm{v} / \mathrm{v})$ Triton $X-100$ in $100 \mu \mathrm{l} 0.1 \%(\mathrm{w} / \mathrm{v})$ sodium citrate for $4 \mathrm{~min}$ in ice. Then, samples were split into two aliquots and subsequently incubated ( $\mathrm{h}$ at room temperature) either with anti-CatSperl antibody ( $4 \mu \mathrm{g} / \mathrm{ml}$, test sample) or rabbit serum $(4 \mu \mathrm{g} / \mathrm{ml}$, the negative control). After being washed twice in I\% NGSPBS, spermatozoa were incubated in the dark for $60 \mathrm{~min}$ with goat anti-rabbit IgG-FITC (dilution I:100 in 1\% NGS-PBS). Samples were washed twice, re-suspended in $300 \mu \mathrm{lPBS}$, stained with $4.5 \mu \mathrm{IPI}(50 \mu \mathrm{g} / \mathrm{ml}$ in PBS) and incubated in the dark for $15 \mathrm{~min}$ at room temperature. An additional aliquot of sperm suspension was prepared with the same procedure as for the test sample but omitting the PI staining and used for instrumental compensation.

Samples were acquired using a fluorescence-activated cell sorter (Becton Dickinson, Mountain View, CA, USA) equipped with a I5-mW argon ion laser for excitation. Green fluorescence of FITC-conjugated goat anti-mouse IgG was revealed by an FL-I (5 I5-555-nm wavelength band) detector; red fluorescence of PI was detected by an FL-2 (563-607-nm wavelength band) detector. We acquired 8000 nucleated events in the gate (i.e. the events labeled with $\mathrm{Pl}$ ) of the characteristic forward scatter/side scatter region of sperm cells (Muratori et al., 2008). A marker was established in the CatSperl axis dot plot of the negative control, including $99 \%$ of total events. This marker was translated in the corresponding test sample and all the events beyond the marker were considered positive for CatSperl. The mean fluorescence intensity (MFI) of CatSperl in sperm was calculated as the ratio between the mean intensity of cells of the test sample and the mean intensity of cells of the corresponding isotype negative control.

To determine CatSperl localization, labeled sperm samples were examined in a fluorescence microscope (Carl Zeiss, Axiolab AI FL, Milan, Italy), equipped with filter set I5, 44 and 49 by an oil immersion $\times 100$ magnification objective. Images were captured by an AxioCam ICm I camera with Axiovision 4.8 (provided by Carl Zeiss, Milan, Italy) and edited by Adobe Photoshop CS 2 (Adobe Systems, Inc., San Jose, CA, USA). Localization of the CatSper I signal was evaluated in 200 spermatozoa from 10 asthenozoospermic and 10 normozoospermic subjects.

Table I Average age and semen characteristics in normozoospermic (N) and asthenozoospermic (A) patients.

\begin{tabular}{|c|c|c|c|}
\hline Parameters & $N(n=85)$ & $A(n=30)$ & $P$ value \\
\hline Age & $34.8 \pm 7.5$ & $38.2 \pm 8.9$ & n.s. \\
\hline Progressive motility (\%) & $57.2 \pm 12.4$ & $20.1 \pm 7.2$ & $<0.001$ \\
\hline Immotile sperm (\%) & $33.7 \pm 11.0$ & $65.1 \pm 11.7$ & $<0.001$ \\
\hline Total motility (\%) & $66.4 \pm 10.8$ & $34.9 \pm 11.7$ & $<0.001$ \\
\hline Hyperactivated motility (\%) & $3.0(0 \div 21)$ & $1.0(0 \div 3)$ & $<0.005$ \\
\hline Sperm concentration $\left(\times 10^{6} / \mathrm{ml}\right)$ & $74.0(8.8 \div 330)$ & $19.3(6.5 \div 138.0)$ & $<0.001$ \\
\hline Sperm number $\left(\times 10^{6} /\right.$ ejaculate $)$ & $299.4(40.7 \div 1067)$ & $56.3(13.6 \div 515.6)$ & $<0.001$ \\
\hline Volume (ml) & $4.0 \pm 3.1$ & $3.4 \pm 1.6$ & n.s. \\
\hline Sperm morphology (\%) & $5.5 \pm 3.5$ & $3.1 \pm 2.5$ & $<0.005$ \\
\hline
\end{tabular}

Sperm number, sperm concentration and hyperactivated motility did not follow a normal distribution and are expressed as median (minimum $\div$ maximum). Statistical comparisons were made using the Mann-Whitney $U$-test. All other parameters follow a normal distribution and are expressed as mean \pm SD. Statistical comparisons were made by the $t$-test. 
For experiments of Catsperl detection in live sperm cells, after being washed with HTF medium, fresh semen samples were incubated for I h at room temperature in $500 \mu \mathrm{l}$ PBS with Live/Dead Fixable Far Red Dead Cell Stain Kit (LI 0 I 20, diluted I: I 0 000), washed and processed as described above and acquired using FACSaria II flow cytometer (BD Biosciences, Franklin Lakes, NJ, USA) equipped with a violet laser, a blue laser and a red laser for excitation, respectively, at 405, 488 and $633 \mathrm{~nm}$ (Muratori et al., 20I5).

\section{Assessment of sperm motility by CASA}

Immediately after $40 \%$ PureSperm selection, sperm motility was analyzed by the CASA system (Hamilton Thorn Research, Beverly, MA, USA) as described previously (Luconi et al., 2005; Tamburrino et al., 20I4) in the 60 samples (including 18 from asthenozoospermic men) which maintained the initial motility characteristics after PureSperm selection (see above). Average path velocity (VAP, $\mu \mathrm{m} / \mathrm{s}$ ), straight line velocity (VSL, $\mu \mathrm{m} / \mathrm{s}$ ), curvilinear velocity $(V C L, \mu \mathrm{m} / \mathrm{s})$, amplitude of lateral head displacement $(A L H$, $\mu \mathrm{m})$, beat cross frequency (BCF, $\mathrm{Hz}$ ), linearity of progression (LIN, \%), percentages of sperm cells with total, progressive and rapid motility (sperm with VAP $\geq 25 \mu \mathrm{m} / \mathrm{s}$ ), percentages of sperm cells with non-progressive motility (static) and hyperactiveted spermatozoa (HA) were recorded. The threshold values for $\mathrm{HA}$ cells identification were manually set $(\mathrm{VCL}>150 \mu \mathrm{m} / \mathrm{s}$, ALH $>7 \mu \mathrm{m}$, LIN <50\%; Mortimer et al., 1998). A minimum of 100 motile cells and 5 fields were analyzed for each aliquot. All analyses were performed at $37^{\circ} \mathrm{C}$.

\section{Evaluation of intracellular calcium concentrations $\left(\left[\mathrm{Ca}^{2+}\right]_{\mathrm{i}}\right)$ in capacitated spermatozoa}

$\left[\mathrm{Ca}^{2+}\right]_{i}$ was evaluated as previously described (Krausz et al., 1995, 1996; Tamburrino et al., 2014) in 40 samples (including 10 from asthenozoospermic men) selected by PureSperm (see above). Briefly, after $40 \%$ PureSperm selection, spermatozoa were capacitated for $3 \mathrm{~h}$ in HTF-10\% HSA medium and incubated for $30 \mathrm{~min}$ at $37^{\circ} \mathrm{C}$ with Fura2/AM for $45 \mathrm{~min}$, washed, resuspended in FM buffer ( $125 \mathrm{mM} \mathrm{NaCl}, 10 \mathrm{mM} \mathrm{KCl}, 2.5 \mathrm{mM} \mathrm{CaCl}_{2}, 0.25 \mathrm{mM}$ $\mathrm{MgCl}_{2}$, $19 \mathrm{mM}$ sodium lactate, $2.5 \mathrm{mM}$ sodium pyruvate, $20 \mathrm{mM}$ HEPES/ $\mathrm{NaOH})$ and further incubated for $30 \mathrm{~min} .\left[\mathrm{Ca}^{2+}\right]_{i}$ was measured by using a spectrofluorimetric method with a Perkin-Elmer LS50B instrument equipped with a fast rotary filter shuttle for alternate 340 and $380 \mathrm{~nm}$ excitation. Spermatozoa were stimulated with progesterone $10 \mu \mathrm{M}$ which was added directly in the cuvette. Fluorescence measurements were converted to $\left[\mathrm{Ca}^{2+}\right]_{\mathrm{i}}$ by determining maximal fluorescence (Fmax) with digitonin $(0.01 \%(w / v)$ final concentration) followed by minimal fluorescence (Fmin) with 10 mM EGTA, pH I0. $\left[\mathrm{Ca}^{2+}\right]_{\mathrm{i}}$ was calculated according to Grynkiewicz et al. (1985) assuming a dissociation constant of Fura 2 for calcium of $224 \mathrm{nM}$. Each sample was evaluated in triplicate and reported results are referred to the mean values of at least two determinations. $\Delta\left[\mathrm{Ca}^{2+}\right]_{i}$ was calculated as the difference between progesterone-stimulated and basal $\left[\mathrm{Ca}^{2+}\right]_{\mathrm{i}}$.

\section{Evaluation of the AR}

AR was evaluated in 35 samples (including 9 from asthenozoospermic men) selected by $40 \%$ PureSperm. Spermatozoa $\left(10 \times 10^{6} / \mathrm{ml}\right)$ were capacitated in $\mathrm{HTF}-10 \% \mathrm{HSA}$ for $3 \mathrm{~h}$ and stimulated for I $\mathrm{h}$ with progesterone $(10 \mu \mathrm{M})$ dissolved in dimethyl sulfoxide (DMSO) at an initial concentration of $0.1 \mathrm{M}$ and diluted in HTF/HSA medium. Control samples were incubated with $\operatorname{DMSO}(0.0001 \%, v / v)$. Sperm AR was evaluated through the use of the fluorescent probe FITC-labeled Arachis hypogea (peanut) lectin as previously described (Falsetti et al., 1993; Krausz et al., 1995; Tamburrino et al., 20I4). In order to evaluate AR only in live spermatozoa, at the end of the incubation period spermatozoa were washed and re-suspended in $0.5 \mathrm{ml}$ of hypo-osmotic swelling medium $(\mathrm{WHO}, 2010)$ for $\mathrm{I} h$ at $37^{\circ} \mathrm{C}$, washed again and finally re-suspended in $50 \mu \mathrm{l}$ ice-cold methanol. The sperm suspension was layered on a slide, air-dried at room temperature and stored at $-20^{\circ} \mathrm{C}$. On the day of evaluation, the slides were stained with FITC-labeled Arachis hypogea (peanut) lectin and fluorescence was observed in a fluorescence microscope. A total of 200 curled-tail (viable) spermatozoa per slide were scored for the AR. The difference between the AR percentage in the progesterone-induced and control was considered to be the percentage of spermatozoa responsive to the progesterone stimulation (acrosome reaction following progesterone challenge, ARPC).

\section{Immunohistochemistry and confocal microscopy}

Double-immunostaining for CatSper I and acrosin was performed on human testicular biopsies as described (Muciaccia et al., 2005, 20 I3). Briefly, surgical testicular samples were obtained from two heart-beating organ donors at the hospital Policlinico Umberto I at the Sapienza University of Rome, Italy. Biopsies were fixed in 10\% ( $\mathrm{v} / \mathrm{v})$ buffered formalin and embedded in paraffin. Three to five- $\mu$ m-thick paraffin sections were serially collected and mounted on polylysine-coated slides. Dewaxed and rehydrated sections were incubated in $10 \mathrm{mM}$ citrate buffer $(\mathrm{pH} 6.0)$ in a microwave oven for antigen retrieval at $750 \mathrm{~W}$, three times for $5 \mathrm{~min}$. After blocking non-specific binding with $5 \%(\mathrm{v} / \mathrm{v}) \mathrm{NGS}$ plus $5 \%(\mathrm{v} / \mathrm{v}) \mathrm{NDS}$, sections were incubated with monoclonal anti-acrosin (diluted $1: 1000$ ) and polyclonal anti-CatSperl (diluted I:100) primary antibodies overnight at $4^{\circ} \mathrm{C}$. After being washed, samples were incubated with secondary antibodies: Alexafluor 488 goat antirabbit (diluted I:1000) and donkey Cy3-conjugated anti-mouse (diluted $\mathrm{l}: 300$ ), for $2 \mathrm{~h}$ at room temperature. Negative control experiments were performed by using rabbit serum instead of primary antibody. Nuclei were stained using TOTO3 dyes ( 1 :2000). Slides were observed with a Leica laser scanning microscope TCS SP2 equipped with three lasers (Leica, Wetzlar, Germany). Each channel was acquired separately by using specific laser lines to avoid a bleed-through of the fluorochromes. Photomicrographs were acquired with LAS AF Leica Confocal Software (Leica, Wetzlar, Germany).

\section{Statistical analysis}

Statistical analysis was performed using the Statistical package for the Social Sciences version 20.0 (SPSS, Chicago, IL, USA) for Windows. The Kolmogorov-Smirnov test was used to test the data distribution. Data are expressed as mean \pm SD when normally distributed and as median with min and max values when non-normally distributed. For non-normally distributed parameters, differences between groups were evaluated by the Mann-Whitney non-parametric tests; for normally distributed parameters unpaired two-sided Student's t-test was used. For normally distributed parameters correlations were assessed by Pearson's correlation tests, whereas for all other parameters Spearman's regression analysis was used. Multiple linear regression analysis was applied whenever appropriate. A P-value of $<0.05$ was considered significant in all the regression and statistical analyses.

A receiver operating characteristic ( $R O C$ ) curve was prepared in order to evaluate the accuracy of the percentage of spermatozoa expressing CatSper I in predicting the occurrence of HA.

\section{Results}

\section{CatSper I protein expression in spermatozoa from normozoospermic and asthenozoospermic men}

The cytometric protocol revealed a significantly different percentage of CatSperl-expressing spermatozoa between asthenozoospermic $(n=$ $30)$ and normozoospermic $(n=85)$ men (respectively, mean \pm SD: 
$53.02 \pm 15.5 \%$, and $67.9 \pm 17.1 \%, P<0.001)$. Similar results were obtained when the MFI of CatSperl expression was considered (data not shown).

\section{CatSperl protein expression and semen parameter values}

To investigate if CatSper I expression was related to testicular function and sperm quality, we correlated the percentage of sperm cells expressing CatSper I with the values of standard semen parameters (univariate analysis). Positive correlations between CatSperl expression and progressive and total motility (Fig. I A and B and Table II), and a negative correlation with the percentage of immotile spermatozoa (Table II) were found. CatSperl expression was also positively associated with sperm concentration and total number/ejaculate, and negatively correlated with patient age (Table II). Similar results were obtained when MFI of CatSperl expression was considered (data not shown). Age was negatively associated with total motility $(r=-0.26, P<0.01)$. After multiple regression analysis including age, sperm concentration and motility, correlations between CatSperl expression and progressive (adjusted $r=$ $0.3, P<0.005$ ) and total (adjusted $r=0.3, P<0.00 \mathrm{I}$ ) motility and age (adjusted $r=-0.18, P<0.05)$ remained significant. Although such correlations were highly significant, a high percentage of spermatozoa expressing CatSper I were immotile (the intercept of the regression line occurs at $\sim 40 \%$ CatSperl expression, Fig. IB). To understand whether such a discrepancy may be due to expression of the channel in dead spermatozoa, we used the probe LI0I20, which binds to dead cells in a stable manner allowing evaluation of CatSperl in both LI0I20-negative (live) and -positive (dead) sperm (Marchiani et al., 20II; Muratori et al., 2015). Although CatSperl was prevalently expressed in live spermatozoa ( $53.3 \pm 14 \%, n=3), 20.4 \pm 0.2 \%$ of dead sperm cells also expressed the protein explaining, at least in part, the occurrence of patients with high CatSperl expression and low motility.
As mentioned, a recent report (Chung et al., 2014) demonstrated that CatSper may be absent or mislocalized in immotile mouse sperm. To evaluate whether CatSper I localization differed between asthenozoospermic and normozoospermic subjects, we evaluated the subcellular localization of anti-CatSperl staining by fluorescent microscopy in spermatozoa from 10 subjects for each category. In some cells, the staining was punctuated and localized exclusively in the principal piece (example in Fig. 2A) while in other cells, the staining was localized in different sperm structures, such as, but not exclusively, the midpiece (examples in Fig. 2B). We next calculated the percentage of cells showing the two different staining patterns in the total CatSper I-positive spermatozoa. We found that the percentage of spermatozoa showing

\section{Table II Correlations between cationic channel of sperm (CatSper) I expression and semen parameter values $(n=I \mid 5)$.}

\begin{tabular}{|c|c|c|}
\hline Parameters & Correlation coefficients & $P$ value \\
\hline Age & -0.25 & $<0.005$ \\
\hline Progressive motility & 0.37 & $<0.001$ \\
\hline Immotile sperm & -0.41 & $<0.001$ \\
\hline Total motility & 0.39 & $<0.001$ \\
\hline In situ motility & -0.09 & n.s. \\
\hline Sperm concentration & 0.25 & $<0.01$ \\
\hline Sperm number & 0.22 & $<0.05$ \\
\hline Volume & -0.05 & n.s \\
\hline Sperm normal morphology & -0.06 & n.s. \\
\hline
\end{tabular}

Correlations were assessed by Pearson's correlation analysis for all parameters except for in situ motility, sperm concentration and sperm number where Spearman's correlation analysis was used.
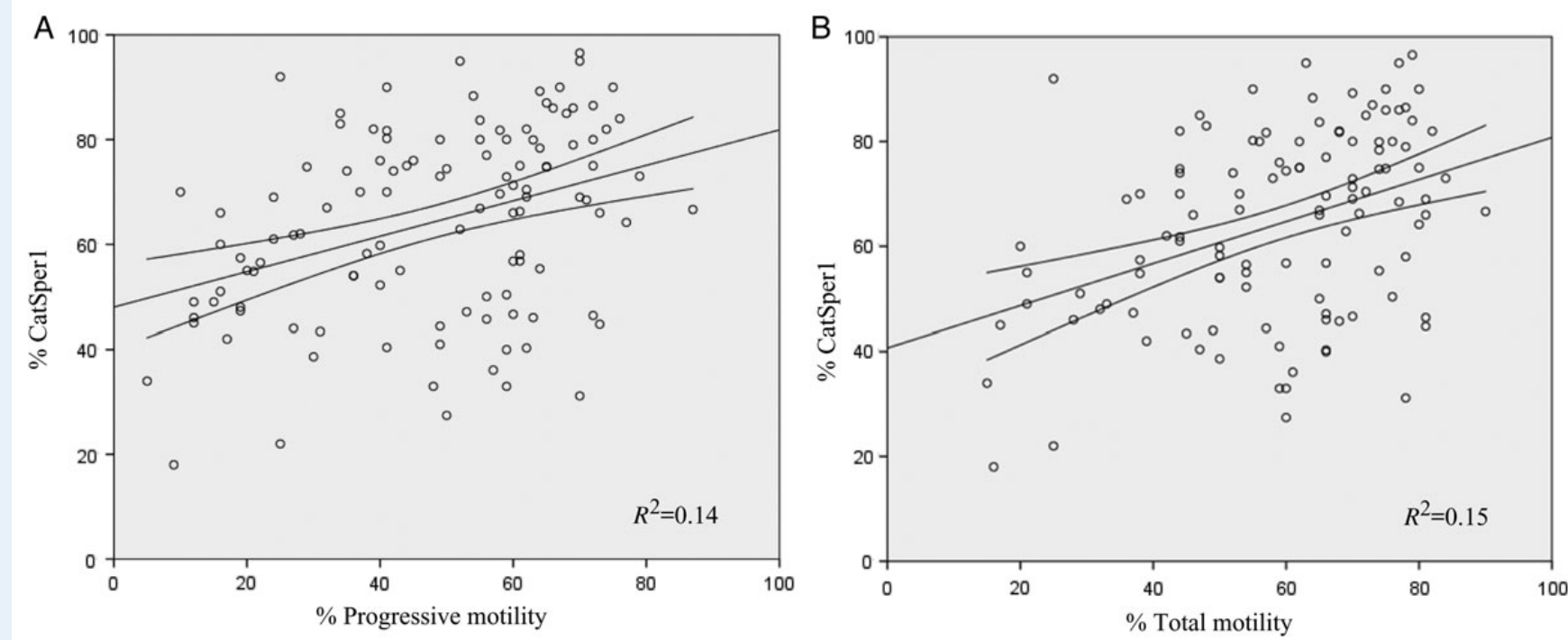

Figure I Scatterplots of correlations between percentages of cationic channel of sperm (CatSper) I-expressing human spermatozoa and progressive (A) and total (B) motility. 95\% Confidence intervals of the correlations and $R^{2}$ values for linear regression analysis are shown. 
A

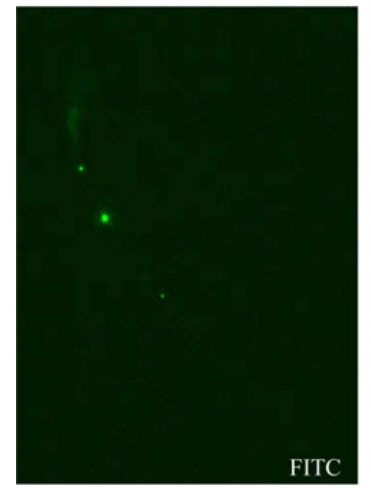

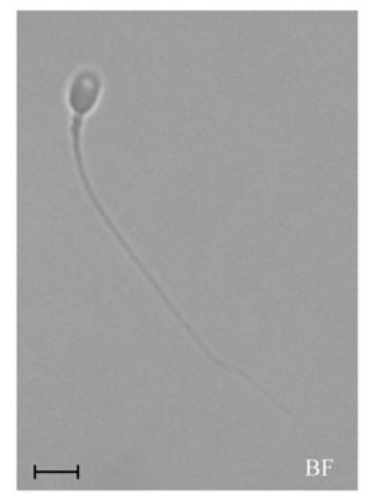

B
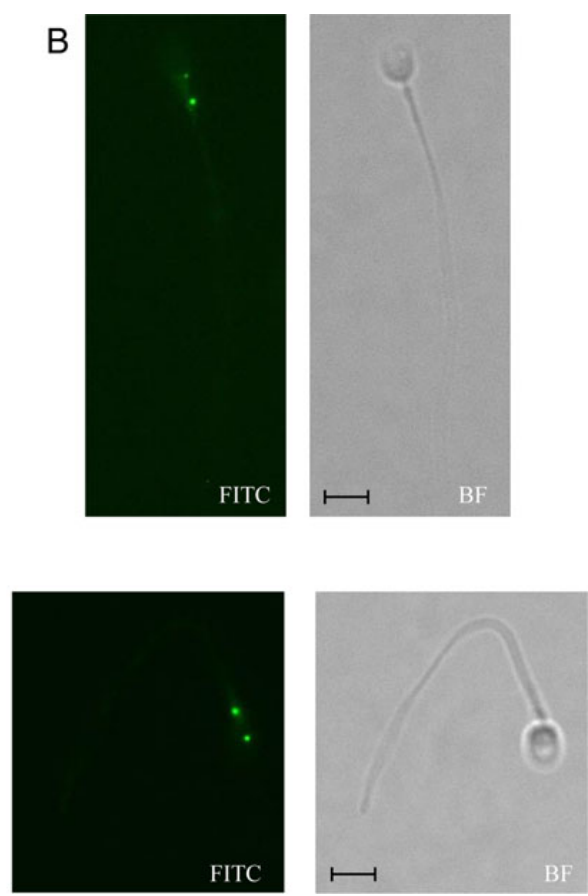

Figure 2 CatSperl localization in human spermatozoa. Representative images ( $\times 1000$ magnification) from fluorescence microscopy showing CatSper I localization in the principal piece (panel A) or in non-canonical sperm structures (panel B). The bright field is shown in adjacent right panels. Scale bar: $5 \mu \mathrm{m}$.

CatSperl exclusively in the principal piece was higher in normozoospermic subjects $(49.9 \pm 7.7 \%$ versus $36.7 \pm 8.7 \%$ in asthenozoospermic subjects, $P<0.05)$.

The occurrence of different patterns of expression of CatSperl in ejaculated spermatozoa prompted us to investigate Catsper I localization in developing human germ cells. Testicular sections obtained from heartbeating donors, where all the steps of germ cell differentiation are present, were co-stained with anti-CatSperI and anti-acrosin antibodies (Fig. 3A). Acrosin detection allows the identification of the different steps of acrosome biogenesis in haploid spermatids (Muciaccia et al., 20I3). Interestingly, CatSperl partially co-localizes with acrosin in round spermatids from step 2 to step 7 (Fig. 3B). In step 8 spermatids, CatSper I immunostaining decreases in the acrosomal region but in favorable sections CatSperl immunostaining was found at the caudal pole of the nucleus close to the base of the developing flagellum (Fig. $3 \mathrm{C}$ ). In elongating spermatids, CatSperl immunostaining in the acrosomal region became undetectable (Fig. 3B, step 10).

\section{CatSperI expression and CASA parameter values}

The relationship between CatSper I and HA was evaluated by measuring sperm motion parameters by CASA in sperm samples from 60 men. CatSperl expression was correlated with most CASA parameter values (Table III and Fig. 4). In particular, VCL (Fig. 4A) and LIN (Fig. 4B), two main parameters defining HA (Mortimer et al., 1998), were, respectively, positively and negatively correlated with CatSper I expression, which was positively correlated with HA (Fig. 4C). A positive correlation was also present between CatSperl expression and percentage total, progressive and rapid motility as evaluated by CASA (Table III).
After adjustment for patient age and sperm concentration, most correlations between CatSper I expression and CASA motility parameters were maintained (Table III).

To determine the accuracy of CatSperl expression in predicting the \% HA of the sample, we used ROC as a binary classifier system. A value of 10\% HA was chosen from the study of Alasmari et al. (2013a) showing that at this threshold HA was able to distinguish between donors and subfertile patients. At a threshold of 75.5\%, CatSperl expression predicted the occurrence of I0\% HA with an accuracy of $83.7 \pm 6.2 \%(P<0.0 \mathrm{I})$ showing a sensitivity and a specificity of 83 and $73 \%$, respectively (Fig. 4D). Conversely, other parameters that can be evaluated by routine semen analysis could not predict achievement of HA status (Table IV).

\section{CatSper I expression and $\left[\mathrm{Ca}^{2+}\right]_{\mathrm{i}}$}

A significant correlation was found between CatSperl expression and progesterone-stimulated $\left[\mathrm{Ca}^{2+}\right]_{\text {i }}$, both in terms of peak concentration $(r=0.36, P<0.05)$ and $\Delta$ increase $(r=0.36, P<0.05, n=40$; Fig. 5). Both correlations were maintained after adjustment for patient age and sperm concentration (progesterone-stimulated $\left[\mathrm{Ca}^{2+}\right]_{\text {, }}$ adjusted $r=0.39, P<0.05 ; \Delta$ increase, adjusted $r=0.42, P<0.01$ ). Conversely, no correlation was found between CatSperl expression and basal $\left[\mathrm{Ca}^{2+}\right]_{\mathrm{i}}(r=0.23, P=0.18)$ although the latter was highly significantly correlated with progesterone-stimulated $\left[\mathrm{Ca}^{2+}\right]_{\mathrm{i}}(r=0.87$, $P<0.001, n=40)$.

\section{CatSperll and the AR}

The involvement of CatSper in the AR was examined by directly investigating the relation of basal and progesterone-stimulated $A R$ with 

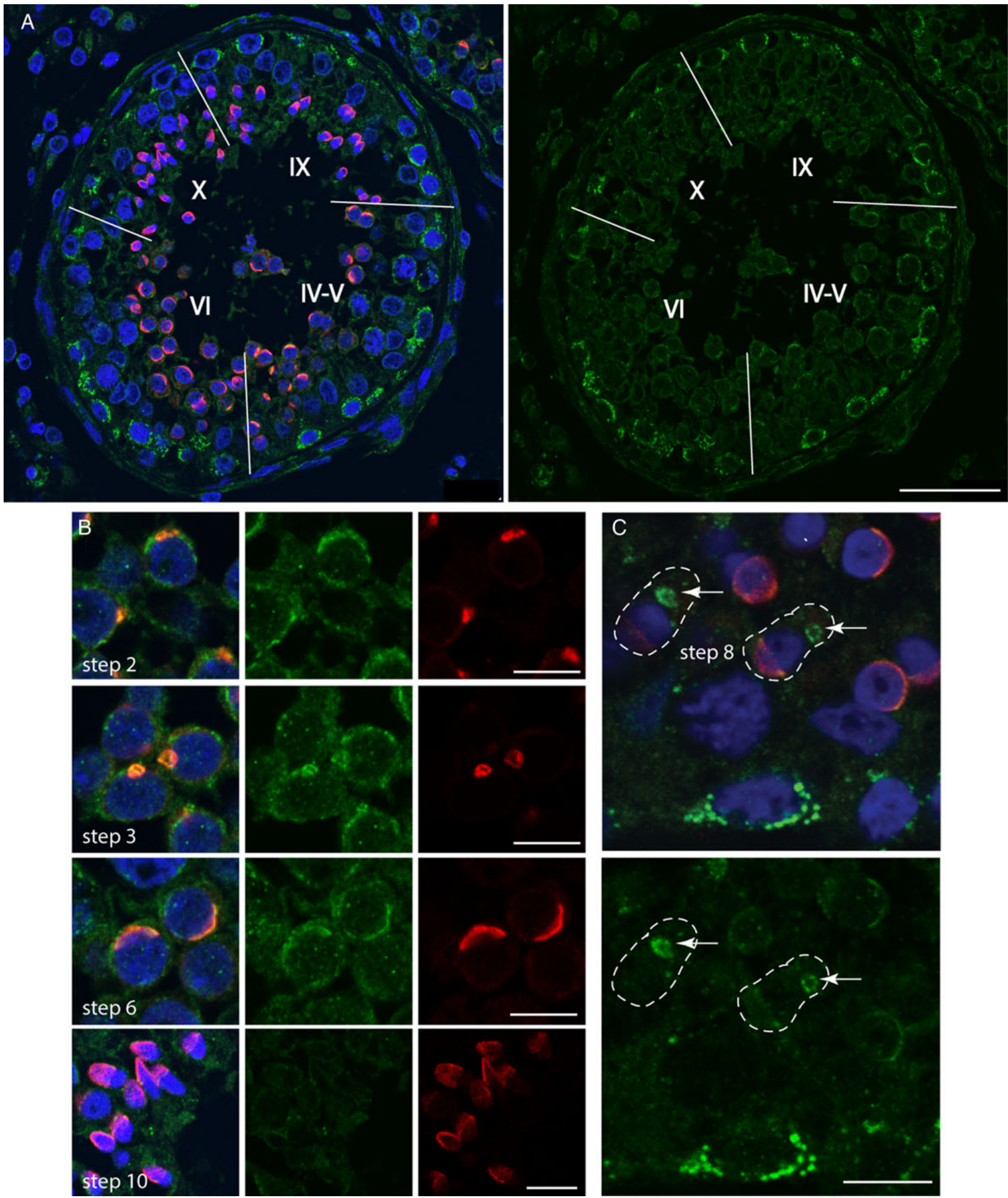

Figure 3 CatSperl protein expression in the human testis. Formalin-fixed paraffin sections were stained with anti-CatSperl (green), anti-acrosin (red) and TOTO-3 (blue, to detect nuclei), and single Z-sections were acquired at the confocal microscope. Merging of the pictures is shown in the left column in $(\mathbf{A})$ and $(\mathbf{B})$ and the upper panel in (C). (A) In the normal human seminiferous epithelium CatSperl expression is high in spermatogonia and decreases in meiotic cells and haploid cells. Roman numerals indicate the germ cell associations of the cycle of the seminiferous epithelium (identification of the stages according to Muciaccia et al. 20I3). (B) In spermatids, CatSperl partially co-localizes with acrosin in round spermatids from step 2 to step 7 (examples of step 2, 3 and 6 are shown) whereas such co-localization is lost from early elongating spermatids and thereafter during spermiogenesis. (C) In step 8 round spermatids, Catsperl localizes at the posterior pole of the nucleus (white arrow) at the base of the developing flagellum. Dashed lines highlight the cellular profiles. Scale bars: $50 \mu \mathrm{m}$ in (A), $10 \mu \mathrm{m}$ in (B) and (C). Pictures are representative of two samples from two testis. 


\begin{tabular}{|c|c|c|c|c|}
\hline CASA parameters & $r$ & $P$ value & Adj. $r$ & Adj. $p$ \\
\hline VAP & 0.29 & $<0.05$ & 0.28 & $<0.05$ \\
\hline VSL & 0.21 & n.s. & 0.19 & n.s. \\
\hline VCL & 0.37 & $<0.00$ I & 037 & $<0.01$ \\
\hline ALH & 0.25 & 0.06 & 0.26 & 0.06 \\
\hline $\mathrm{BCF}$ & 0.17 & n.s. & 0.15 & n.s. \\
\hline LIN & -0.27 & $<0.05$ & -0.29 & $<0.05$ \\
\hline $\mathrm{HA}$ & 0.41 & $<0.05$ & 0.44 & $<0.00$ \\
\hline Total motility & 0.35 & $<0.01$ & 0.33 & $<0.05$ \\
\hline Progressive motility & 0.36 & $<0.01$ & 0.24 & n.s. \\
\hline Rapid & 0.34 & $<0.01$ & 0.32 & $<0.05$ \\
\hline Static motility & -0.35 & $<0.01$ & -0.32 & $<0.05$ \\
\hline
\end{tabular}

Correlations were assessed by Pearson's correlation analysis for all parameters except for progressive, static motility and HA motility where univariate Spearman's regression analysis was used. Adj $r$ and Adj $p$ are, respectively, correlation coefficient and $P$ value adjusted for sperm concentration and patient age.

VAP, average path velocity; VSL, straight line velocity; VCL, curvilinear velocity; ALH, amplitude of lateral head displacement; BCF, beat cross frequency; LIN, linearity of progression.

CatSperl protein expression in 35 subjects. No correlation was found between CatSperl expression and either basal $(r=-22, P=0.19)$ or progesterone-induced AR $(r=-0.14, P=0.4)$ and ARPC $(r=0.08$, $P=0.6)$. In the 32 samples where $\left[\mathrm{Ca}^{2+}\right]_{i}$ and AR were concomitantly evaluated, a significant correlation was found between the percentage of progesterone-stimulated $\mathrm{AR}$ and progesterone-stimulated $\left[\mathrm{Ca}^{2+}\right]_{i}$ $(r=0.49, P<0.01)$. Conversely, basal AR did not show any correlation with basal $\left[\mathrm{Ca}^{2+}\right]_{\mathrm{i}}(r=0.29, P=0.14)$.

\section{Discussion}

The role of CatSper in human sperm pathophysiology is still unclear. Most of our current knowledge derives from studies where CatSper activation was obtained after stimulation with progesterone (Lishko et al., $20 \mathrm{I}$ ), with stimuli increasing intracellular $\mathrm{pH}$ (such as 4-AP) (Alasmari et al., 20I3b) or with low specificity CatSper antagonists (Strunker et al., 20II; Sagare-Patil et al., 2013; Tamburrino et al., 20l4). However, no studies so far have attempted to evaluate a direct relationship between CatSper expression and sperm functions. Here, by employing a large sample size we elucidated the relationship between CatSperl protein expression and the main sperm functions where the channel has been hypothesized to be involved.

In our study, expression of the CatSperl subunit has been assumed to be a measure of the occurrence of the CatSper channel in spermatozoa. Indeed, although functional CatSper channel is formed by four homologous subunits (CatSper $1-4$ ) and at least three auxiliary subunits (CatSper $\beta, \gamma$ and $\delta$ ) (Ren and Xia 20 I0; Chung et al., 20I I), studies in the mouse demonstrate that lack of even one of the CatSper subunits leads to complete absence of the channel in mature sperm (Qi et al., 2007). Studies in men with a CatSper2 gene deletion (Smith et al., 2013), demonstrating lack of CatSper currents and of expression of the CatSper $\beta$ subunit in their sperm, suggest that all subunits are required for channel expression and its function also in humans.

The lower percentage of spermatozoa expressing CatSperl in asthenozoospermic patients and the positive correlations between expression of the channel subunit and motility clearly indicate an involvement of CatSper in the pathogenesis of asthenozoospermia, as previously suggested (Nikpoor et al., 2004; Li et al., 2007; Bhilawadikar et al., 20 I3; Tamburrino et al., 20I4). Since progressive motility is fundamental for penetration of the cervical barrier, our results highlight the importance of CatSper in this process (Alasmari et al., 2013b). The reason for lower expression of CatSperl in asthenozoospermic men and nonmotile spermatozoa is presently unclear but may be related to disturbances in spermatogenesis leading to anomalies in the production or increased degradation of the proteins forming the channel, or their assembly. The recent identification of heterozygous mutations of CatSperl-4 genes in 6 of 30 men with asthenozoospermia (which have not been found in normozoospermic men) (Visser et al., 20l I) suggests that alterations in channel expression or function may also be due to mutations. Moreover, we show here that CatSperl protein is predominant in live spermatozoa (as only $20 \%$ of dead ones express the channel), suggesting that processes, such as apoptosis, leading to sperm death may also result in a lack of expression, degradation or incorrect assembly of the channel. On the other hand, the occurrence of CatSperl in $20 \%$ of dead spermatozoa might explain, at least in part, the subjects with high CatSperl expression and low sperm motility. However, sperm motility is highly influenced by several other factors (for example oxidative stress producing membrane damage and altered mitochondrial function), which could explain reduced motility in spite of an elevated percentage of CatSperl-expressing spermatozoa. Furthermore, it has recently been shown that in non-motile mouse spermatozoa, CatSper domains may not only be degraded but also are no longer confined to the principal piece of the tail (Chung et al., 20I4). Here, by examining CatSper I localization in a large number of spermatozoa from asthenozoospermic and normozoospermic men, we found that although CatSper I was mostly expressed in the principal piece, non-canonical localization of the protein was also observed and this was higher in asthenozoospermic men. This result indicates that, besides a lower percentage of sperm cells expressing CatSper I, patients with low sperm motility may also display a non-functional localization. Indeed, at least in mouse spermatozoa, such incorrect localization does not allow CatSpermediated signaling and tubule assembly necessary for motility (Chung et al., 20|4). The reason for incorrect localization of CatSper signal is presently unclear. We show here, by immunofluorescence confocal analysis, that during the last phases of sperm differentiation, CatSperl expression moves from the head to the base of the flagellum in testicular specimens with ongoing spermatogenesis. It can be hypothesized that abnormalities during spermiogenesis may lead to a lack or defective reorganization of CatSperl resulting in a malfunctioning and low motility spermatozoon.

Our study also reveals the presence of subjects with low CatSper I expression and elevated motility. In the seven men with CatSper genes deletion so far reported in the literature (reviewed in Hildebrand et al., 2010), sperm motility ranged from 0 to $50 \%$. Clearly, these results suggest that alternative pathways may be activated in spermatozoa that rescue sperm motility in the absence of CatSper.

We found a negative correlation between CatSperl expression and the patients' age, sperm concentration and total sperm number, the 


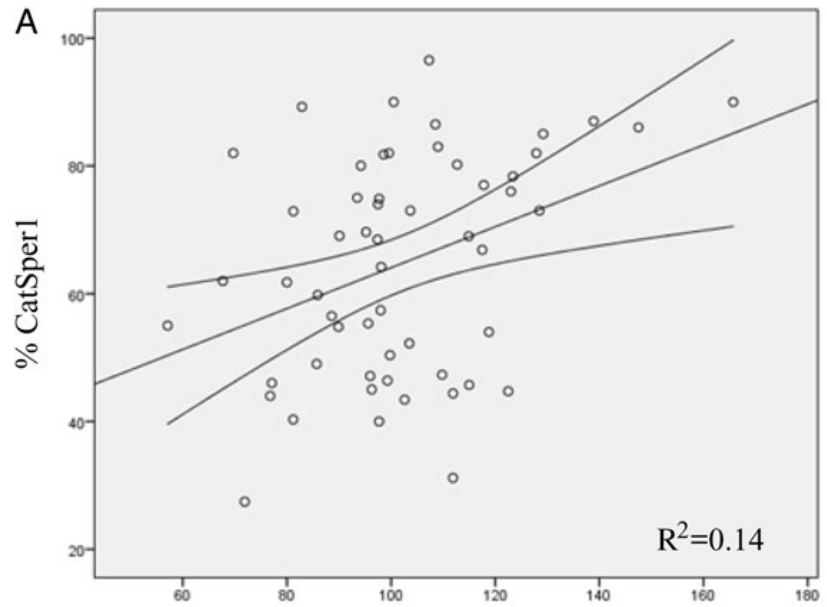

$\operatorname{VCL}(\mu \mathrm{m} / \mathrm{s})$

C

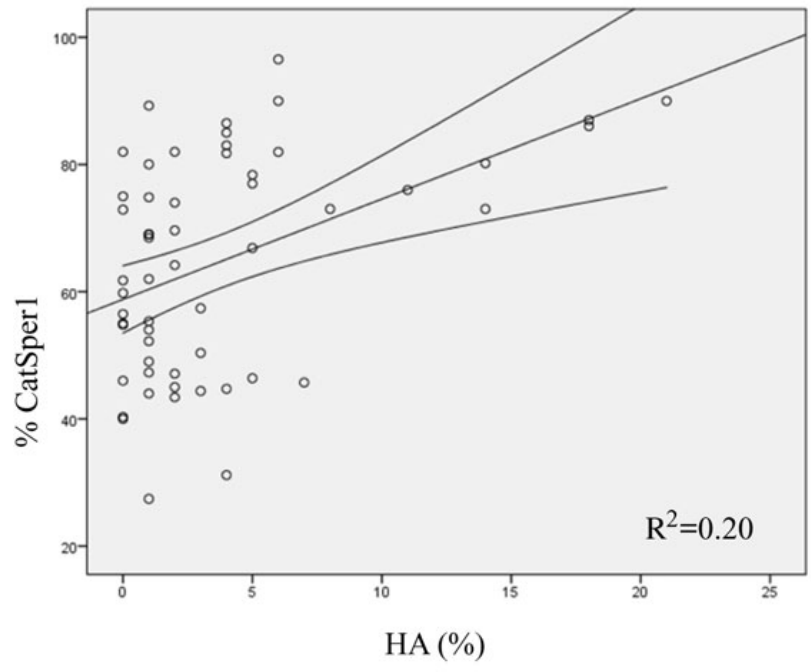

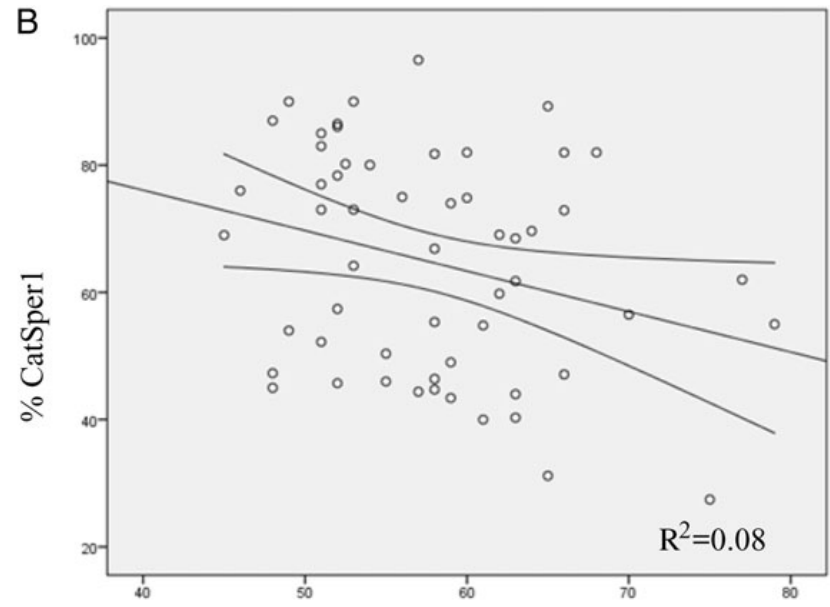

$\operatorname{LIN}(\%)$

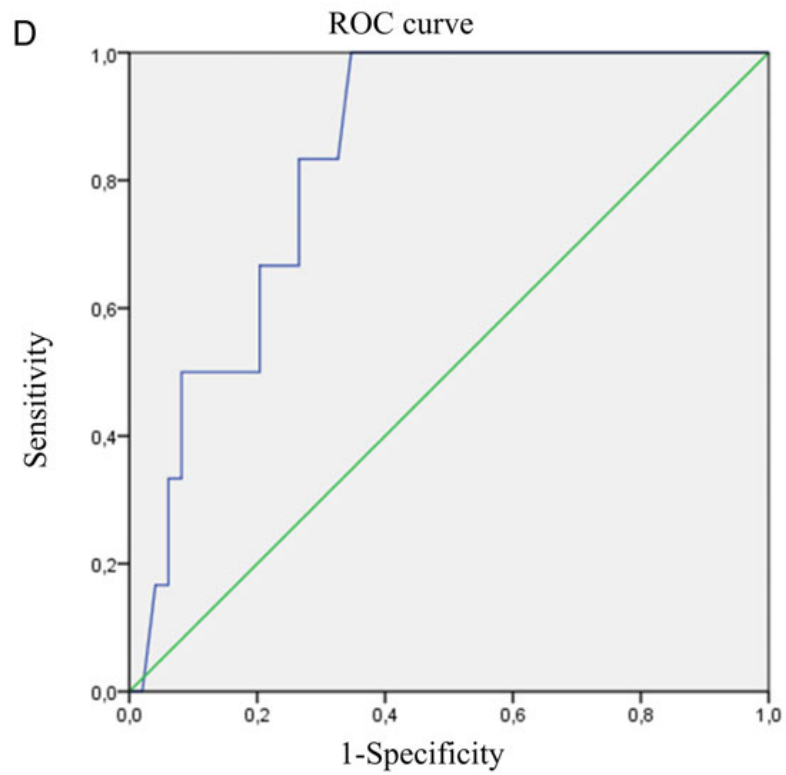

Figure 4 Scatterplots of correlations between percentages of CatSper I-expressing human spermatozoa and curvilinearvelocity (VCL, panel A), linearity of progression (LIN, panel B) and hyperactivation (HA, panel C) as assessed by CASA. 95\% Confidence intervals of the correlations (upper and lower lines) and $R^{2}$ values for linear regression analysis are shown. (Panel D) ROC curve of the ability of CatSper I protein expression to predict I $0 \% \mathrm{HA}$. The area under the ROC curve is 0.83 . The optimal cutoff for the prediction of $\mathrm{HA}$ is $75.5 \%$, with $83 \%$ sensitivity and $73 \%$ specificity.

\section{Table IV ROC curve analysis of the accuracy of routine semen parameters in predicting $\mathrm{HA} \geq 10 \%$.}

\begin{tabular}{|c|c|c|}
\hline Semen parameters & AUC (\% \pm SE) & $P$ value \\
\hline Progressive motility & $61 \pm 9.5$ & n.s. \\
\hline Total motility & $61 \pm 9.3$ & n.s. \\
\hline Sperm concentration & $72 \pm 9.0$ & n.s. \\
\hline Sperm number & $72 \pm 8.1$ & n.s. \\
\hline Morphology & $49 \pm 14$ & n.s. \\
\hline
\end{tabular}

A threshold value of $10 \%$ HA was chosen on the basis of the study of Alasmari et al. (2013a).

AUC, area under the curve, percentage value. latter two consistent with the frequent oligozoospermic phenotype observed in CatSper-deleted men (Hildebrand et al., 2010). However, after multiple linear regression analysis including progressive sperm motility, patients' age and sperm concentration or number, only age and motility maintained significant correlations, indicating that correlations with sperm number and concentration are biologically irrelevant. A negative correlation of CatSperl with patients' age suggests that mechanisms of regulation of its expression in the testis might be altered with aging. Although a decrease in human semen quality with age is highly debated (Bujan et al., 1996; Kidd et al., 200 I; Eskenazi et al., 2003), in our particular cohort sperm motility was negatively associated with age. Aging may cause degenerative alterations in the germinal epithelium affecting sperm quality (Kidd et al., 200 I). We speculate that such alterations may also affect CatSperl expression. The CatSperl gene promoter contains 


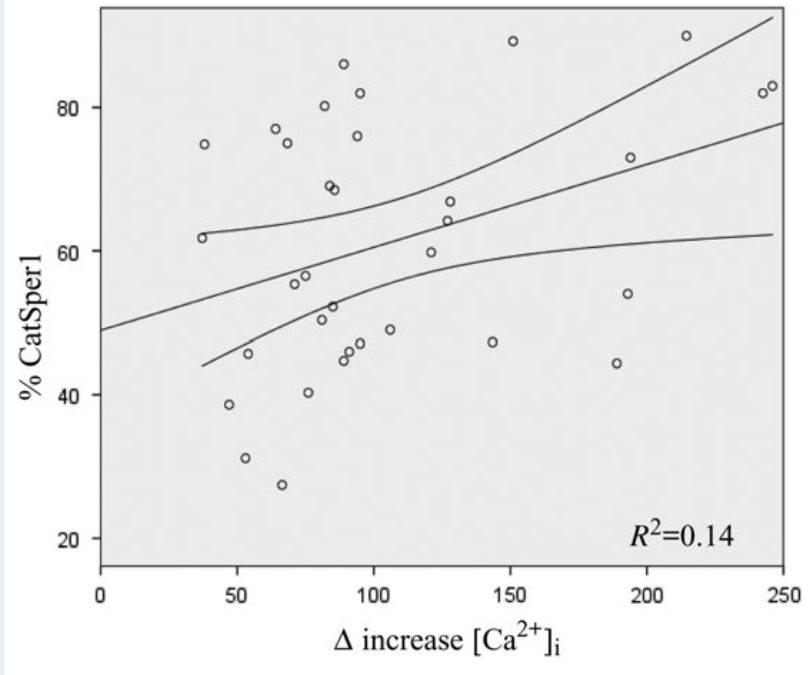

Figure 5 Scatterplot of correlation between percentages of CatSperl-expressing human spermatozoa and $\Delta\left[\mathrm{Ca}^{2+}\right]_{\mathrm{i}} . \Delta\left[\mathrm{Ca}^{2+}\right]_{\mathrm{i}}$ was calculated as the difference between progesterone-stimulated and basal $\left[\mathrm{Ca}^{2+}\right]_{\mathrm{i}}$. $95 \%$ Confidence intervals of the correlations and $R^{2}$ values for linear regression analysis are shown.

multiple binding sites for transcription factors (Mata-Rocha et al., 20I3, 20I4) including members of the SOX gene family of fundamental importance for testicular development and male fertility (Jiang et al., 2013). Studies in rodents demonstrate that CatSper gene expression is increased by agents such as selenium and Vitamin $\mathrm{E}$ (Mohammadi et al., 2009, 2013) and modulated by drugs that affect sperm quality (Wang et al., 2013; Amini et al., 20l4; Park et al., 2014), suggesting that expression of the channel is regulated in the testis by oxidative status and lifestyle habits. It can be speculated that such factors are involved in down-regulating CatSperl expression in the aging male and in men with asthenozoospermia.

Whereas studies in CatSperknockout (KO) mice show a clear involvement of the channel in sperm HA, studies in men are less clear on this. Alasmari et al. $(2013 a, b)$ reported that CatSper activation was not sufficient to achieve $\mathrm{HA}$ and that the failure of $\mathrm{HA}$ in response to stimulation was more common in IVF patients than in donors. Similarly, after stimulation with progesterone, we detected an increase in HA in only a few subjects (Tamburrino et al., 20I4). Here, we found a significant correlation between CatSperl expression and basal (spontaneous) HA levels, which indicates an involvement of the channel in the process of the development of HA. Such a finding is not surprising in view of the role of calcium in the HA process (Suarez, 2008) and suggests that failure to achieve HA in sub-fertile subjects may be related to poor, absent or mislocalized expression of the channel on spermatozoa. Importantly, we show here that the percentage of spermatozoa expressing CatSperl (but none of the parameters evaluated by routine semen analysis) predicts with high accuracy, sensitivity and good specificity, the ability of the sample to reach a level of $\mathrm{HA}$ of $10 \%$ which distinguishes between donors (young men with no known fertility problems) and subfertile subjects (Alasmari et al., 2013a), reinforcing involvement of the CatSper channel in establishment of HA in human spermatozoa. HA is related to natural fertility (Peedicayil et al., 1997; Mazzilli et al., 200I) and predicts results of IVF (Wang et al., 1993; Sukcharoen et al., 1995; Hirano etal., 200 I). Conversely, routine semen analysis is poorly predictive of both natural and in vitro fertilization (Liu and Baker, 2002; Leushuis et al., 20I4). Further studies are needed to understand the relation of sperm CatSper expression with natural and assisted fertilization.

As expected, by evaluating the relationship between CatSperl expression and basal and progesterone-stimulated $\left[\mathrm{Ca}^{2+}\right]_{\mathrm{i}}$ accumulation, a positive correlation was found with the initial peak of $\left[\mathrm{Ca}^{2+}\right]_{i}$ in response to the steroid. However, the correlation with progesterone response was not as tight as expected if CatSper were the only pathway involved in the process. On the other hand, the $\left[\mathrm{Ca}^{2+}\right]_{i}$ in response to the steroid is not totally blunted by CatSper inhibitors (Strunker et al., 20II) even at concentrations higher than those that completely blocked the CatSper current (Lishko et al., 20 I I), suggesting involvement of other pathways (Publicover and Barratt, 20I I). Furthermore, in our hands, no correlation was found between CatSperl expression and basal $\mathrm{Ca}^{2+}$, suggesting that $\mathrm{CatSper} \mathrm{is} \mathrm{not} \mathrm{the} \mathrm{main} \mathrm{player} \mathrm{for} \mathrm{establishing}$ or maintaining $\left[\mathrm{Ca}^{2+}\right]_{\mathrm{i}}$ in unstimulated conditions. By evaluating CatSper currents, Lishko et al. (20l I) demonstrated that human sperm currents were overall smaller and reached similar values to those of the mouse only after stimulation with progesterone, indicating low channel activation in basal conditions. Conversely, in mouse sperm progesterone is not effective, underlying a striking difference between CatSper activation in the two species (Miller et al., 20 I4). Most likely, release of $\mathrm{Ca}^{2+}$ from intracellular stores located in the neck and in the acrosome (Publicover and Barratt, 20 II) or other pathways or channels located in the membrane are involved in maintaining basal calcium in human spermatozoa (Marquez et al., 2007; Darszon et al., 20I I). On the other hand, considering the different lengths of the sperm journey in the female genital tracts of the mouse and human, a high activation of CatSper, which may lead to excessive $\mathrm{HA}$, might be unfavorable thus requiring a further activation in the proximity of the oocyte.

We found no correlation between the percentage of CatSperlexpressing spermatozoa and either basal or stimulated AR, in contrast with previous results (Sagare-Patil et al., 2012; Tamburrino et al., 20I4) showing a significant, but incomplete, effect of CatSper inhibition on progesterone-stimulated AR. Moreover the inhibitors themselves showed an effect per se on the AR (Tamburrino et al., 20I4). Lack of correlation between $A R$ and CatSperl expression is in agreement with results in CatSper $\mathrm{KO}$ mouse spermatozoa, showing no alterations of either basal or ionophore-stimulated AR (Xia et al., 2007). In addition, the location of CatSperl in the tail (Smith et al., 2013; Tamburrino et al., 20 I4) and lack of co-localization with acrosin in late phases of spermiogenesis, where immunostaining for CatSperl is detected in the region of the caudal pole of the nucleus, close to the base of the developing flagellum (present study), also exclude a direct involvement of the channel in the AR. However this result appears to contrast with the fact that progesterone-stimulated AR is highly dependent on the increase of $\left[\mathrm{Ca}^{2+}\right]_{i}$ induced by the steroid (Baldi et al., 2009) and that such an increase shows significant associations both with CatSper I expression and progesterone-stimulated AR (this study, Krausz et al., 1995). To reconcile these apparently contrasting results it has recently been demonstrated (Sanchez-Cardenas et al., 20l4) by concomitant evaluation of $A R$ and $\left[\mathrm{Ca}^{2+}\right]_{i}$ modifications in live human spermatozoa that the initial calcium peak in response to progesterone is not sufficient to trigger the AR. Indeed, in most sperm cells undergoing AR in response to the steroid (representing $\sim 12 \%$ of the total number, as in our 
study) the initial peak is followed by a second peak occurring about 10 min later that immediately precedes AR initiation. The nature and the mechanisms of this second peak of $\left[\mathrm{Ca}^{2+}\right]_{i}$ induced by progesterone is unclear. One hypothesis is that it might be related to influx of $\mathrm{Ca}^{2+}$ from fusion pores generated during AR initiation (Sanchez-Cardenas et al., 2014). Clearly, such a mechanism, if involved, would be CatSperindependent, as also suggested by the lack of correlation between $A R$ and CatSperl expression in this study.

One limitation of our study is the fact that CASA motility, AR and $\left[\mathrm{Ca}^{2+}\right]_{\mathrm{i}}$ were evaluated after $40 \%$ PureSperm selection of the samples, possibly not reflecting the whole sperm sample. However, all the samples were carefully evaluated by microscopy before performing the experiments and only those samples where initial motility characteristics were maintained after selection were kept, as indicated by the significant relationship between pre- and post-selection motility.

In conclusion, our study demonstrates a clear implication of the CatSper channel in progressive and HA motility of human spermatozoa and provides preliminary evidence that lack of expression or mislocalization of CatSperl in spermatozoa may be involved in the pathogenesis of asthenozoospermia. Finally, our data reinforce the concept that the CatSper channel represents a possible target for the development of new human contraceptives.

\section{Acknowledgements}

We thank Drs Erminio Filimberti, Selene Degl'Innocenti and Maria Grazia Fino (AOUC Careggi) for semen analysis. We thank Dr Roberto Zonefrati (Department of Surgery and Translational Medicine, University of Florence) for help with calcium experiments.

\section{Authors' roles}

L.T. performed the experiments, collected and analyzed the data and was responsible for manuscript preparation. S.M. was involved in flow cytometry analysis. E.V. and B.M. performed immunofluorescence experiment and confocal microscopy analysis, helped in the interpretation of results. M.C. contributed to set up and perform experiments in live and dead spermatozoa. S.P. contributed in patient enrollment. G.F. contributed to the final review of the manuscript. M.M. was involved in the interpretation of flow cytometric data and helped in the statistical analysis. E.B. designed and coordinated the study and wrote the manuscript. All the authors made substantial contributions in critically revising the article.

\section{Funding}

The study was supported by grants from Italian Ministry of University and Scientific Research (PRIN project to E.B. and FIRB to S.M.) and Regione Toscana (to G.F.).

\section{Conflict of interest}

The authors declare that there is no conflict of interest that could be perceived as prejudicing the impartiality of the research reported.

\section{References}

Alasmari W, Barratt CL, Publicover SJ, Whalley KM, Foster E, Kay V, Martins da Silva S, Oxenham SK. The clinical significance of calcium-signalling pathways mediating human sperm hyperactivation. Hum Reprod 2013a; 28:866-876.

Alasmari W, Costello S, Correia J, Oxenham SK, Morris J, Fernandes L, Ramalho-Santos J, Kirkman-Brown J, Michelangeli F, Publicover $\mathrm{S}$ et al. $\mathrm{Ca}^{2+}$ signals generated by CatSper and $\mathrm{Ca}^{2+}$ stores regulate different behaviors in human sperm. J Biol Chem 20I3b;288:6248-6258.

Amini M, Shirinbayan P, Behnam B, Roghani M, Farhoudian A, Joghataei MT, Koruji M. Correlation between expression of CatSper family and sperm profiles in the adult mouse testis following Iranian Kerack abuse. Andrology 2014;2:386-393.

Avenarius MR, Hildebrand MS, Zhang Y, Meyer NC, Smith LL, Kahrizi K, Najmabadi H, Smith RJ. Human male infertility caused by mutations in the CATSPERI channel protein. Am J Hum Genet 2009;84:505-5I0.

Avidan N, Tamary H, Dgany O, Cattan D, Pariente A, Thulliez M, Borot N, Moati L, Barthelme A, Shalmon L et al. CATSPER2, a human autosomal nonsyndromic male infertility gene. Eur J Hum Genet 2003; I I:497-502.

Baldi E, Luconi M, Muratori M, Marchiani S, Tamburrino L, Forti G. Nongenomic activation of spermatozoa by steroid hormones: facts and fictions. Mol Cell Endocrinol 2009;308:39-46.

Baldi E, Luconi M, Krausz C, Forti G. Progesterone and spermatozoa: a long-lasting liaison comes to definition. Hum Reprod 20 I I;26:2933-2934.

Bhilawadikar R, Zaveri K, Mukadam L, Naik S, Kamble K, Modi D, Hinduja I. Levels of Tektin 2 and CatSper 2 in normozoospermic and oligoasthenozoospermic men and its association with motility, fertilization rate, embryo quality and pregnancy rate. J Assist Reprod Genet 2013;30:5 I 3-523.

Brenker C, Goodwin N, Weyand I, Kashikar ND, Naruse M, Krähling M, Müller A, Kaupp UB, Strünker T. The CatSper channel: a polymodal chemosensor in human sperm. EMBO J 20 12;3 I: 1654- 1665.

Bujan L, Mansat A, Pontonnier F, Mieusset R. Time series analysis of sperm concentration in fertile men in Toulouse, France between 1977 and 1992. BMJ 1996;3 I 2:47I-472.

Carlson AE, Westenbroek RE, Quill T, Ren D, Clapham DE, Hille B, Garbers DL, Babcock DF. CatSperl required for evoked $\mathrm{Ca}^{2+}$ entry and control of flagellar function in sperm. Proc Natl Acad Sci USA 2003; 100: | $4864-$ | 4868 .

Chung JJ, Navarro B, Krapivinsky G, Krapivinsky L, Clapham DE. A novel gene required for male fertility and functional CATSPER channel formation in spermatozoa. Nat Commun 20 I I;2:153.

Chung JJ, Shim SH, Everley RA, Gygi SP, Zhuang X, Clapham DE. Structurally distinct $\mathrm{Ca}(2+)$ signaling domains of sperm flagella orchestrate tyrosine phosphorylation and motility. Cell 20 I4; I57:808-822.

Darszon A, Nishigaki T, Beltran C, Treviño CL. Calcium channels in the development, maturation, and function of spermatozoa. Physiol Rev 201 1;91:1305-1355.

ESHRE Andrology Special Interest Group. Guidelines on the application of CASA technology in the analysis of spermatozoa. Hum Reprod 1998; I3: 142- | 45 .

Eskenazi B, Wyrobek AJ, Sloter E, Kidd SA, Moore L, Young S, Moore D. The association of age and semen quality in healthy men. Hum Reprod 2003; 18:447-454.

Falsetti C, Baldi E, Krausz C, Casano R, Failli P, Forti G. Decreased responsiveness to progesterone of spermatozoa in oligozoospermic patients. J Androl 1993; 14: 17-22.

Grynkiewicz G, Poenie M, Tsien RY. A generation of Ca indicator with greatly improved fluorescence properties. J Biol Chem 1985;260:3440-3450.

Hildebrand MS, Avenarius MR, Fellous M, Zhang Y, Meyer NC, Auer J, Serres C, Kahrizi K, Najmabadi H, Beckmann JS et al. Genetic male 
infertility and mutation of CATSPER ion channels. Eur J Hum Genet 20 I0; 18: | |78- | | 84.

Hirano Y, Shibahara H, Obara H, Suzuki T, Takamizawa S, Yamaguchi C, Tsunoda H, Sato I. Relationships between sperm motility characteristics assessed by the computer-aided sperm analysis (CASA) and fertilization rates in vitro. J Assist Reprod Genet 200 I; 18:2।3-218.

Jacob A, Hurley I, Mandel FS, Hershlag A, Cooper GW, Benoff S. Human sperm non-nuclear progesterone receptor expression is a novel marker for fertilization outcome. Mol Hum Reprod 1998;4:533-542.

Jaiswal D, Singh V, Dwivedi US, Trivedi S, Singh K. Chromosome microarray analysis: a case report of infertile brothers with CATSPER gene deletion. Gene 20|4;542:263-265.

Jiang T, Hou CC, She ZY, Yang WX. The SOX gene family: function and regulation in testis determination and male fertility maintenance. Mol Biol Rep 2013;40:2187-2194.

Jin J, Jin N, Zheng H, Ro S, Tafolla D, Sanders KM, Yan W. Catsper3 and Catsper4 are essential for sperm hyperactivated motility and male fertility in the mouse. Biol Reprod 2007;77:37-44.

Kidd SA, Eskenazi B, Wyrobek AJ. Effects of male age on semen quality and fertility: a review of the literature. Fertil Steril 200 I;75:237-248.

Kirichok Y, Navarro B, Clapham DE. Whole-cell patch-clamp measurements of spermatozoa reveal an alkaline-activated $\mathrm{Ca}^{2+}$ channel. Nature 2006; 439:737-740.

Krausz C, Bonaccorsi L, Luconi M, Fuzzi B, Criscuoli L, Pellegrini S, Forti G, Baldi $E$. Intracellular calcium increase and acrosome reaction in response to progesterone in human spermatozoa are correlated with in-vitro fertilization. Hum Reprod 1995; 10: 120-124.

Krausz C, Bonaccorsi L, Maggio P, Luconi M, Criscuoli L, Fuzzi B, Pellegrini S, Forti G, Baldi E. Two functional assays of sperm responsiveness to progesterone and their predictive values in in-vitro fertilization. Hum Reprod 1996; I I:1661-1667.

Leushuis E, van der Steeg JW, Steures P, Repping S, Bossuyt PM, Mol BW, Hompes PG, van der Veen F. Semen analysis and prediction of natural conception. Hum Reprod 2014;29:1360-1367.

Li HG, Ding XF, Liao AH, Kong XB, Xiong CL. Expression of CatSper family transcripts in the mouse testis during post-natal development and human ejaculated spermatozoa: relationship to sperm motility. Mol Hum Reprod 2007; 1 3:299-306.

Lishko PV, Botchkina IL, Kirichok Y. Progesterone activates the principal $\mathrm{Ca}^{2+}$ channel of human sperm. Nature 201 I;47 I:387-391.

Liu DY, Baker HW. Evaluation and assessment of semen for IVF/ICSI. Asian J Androl 2002;4:28I-485.

Luconi M, Porazzi I, Ferruzzi P, Marchiani S, Forti G, Baldi E. Tyrosine phosphorylation of the a kinase anchoring protein 3 (AKAP3) and soluble adenylate cyclase are involved in the increase of human sperm motility by bicarbonate. Biol Reprod 2005;72:22-32.

Mansell SA, Publicover SJ, Barratt CL, Wilson SM. Patch clamp studies of human sperm under physiological ionic conditions reveal three functionally and pharmacologically distinct cation channels. Mol Hum Reprod 2014;20:392-408.

Marchiani S, Tamburrino L, Giuliano L, Nosi D, Sarli V, Gandini L, Piomboni P, Belmonte G, Forti G, Baldi E et al. Sumol-ylation of human spermatozoa and its relationship with semen quality. Int J Androl 2011;34:581-593.

Marquez B, Ignotz G, Suarez SS. Contributions of extracellular and intracellular $\mathrm{Ca}^{2+}$ to regulation of sperm motility: release of intracellular stores can hyperactivate CatSperl and CatSper2 null sperm. Dev Biol 2007;303:214-221.

Mata-Rocha M, Alvarado-Cuevas E, Hernández-Sánchez J, Cerecedo D, Felix R, Hernández-Reyes A, Tesoro-Cruz E, Oviedo N. Molecular cloning and analysis of the Catsperl gene promoter. Mol Hum Reprod 2013;19:336-347.
Mata-Rocha M, Hernández-Sánchez J, Guarneros G, de la Chesnaye E, Sánchez-Tusié AA, Treviño CL, Felix R, Oviedo N. The transcription factors Sox5 and Sox9 regulate Catsperl gene expression. FEBS Lett 2014;588:3352-3360.

Mazzilli F, Rossi T, Delfino M, Dondero F, Makler A. A new objective method for scoring human sperm hyperactivation based on head axis angle deviation. Int J Androl 200 I;24: 189-196.

Miki K, Clapham DE. Rheotaxis guides mammalian sperm. Curr Biol 2013; 23:443-452.

Miller MR, Mansell SA, Meyers SA, Lishko PV. Flagellar ion channels of sperm: similarities and differences between species. Cell Calcium 2014 [Epub ahead of print].

Mohammadi S, Movahedin M, Mowla SJ. Up-regulation of CatSper genes family by selenium. Reprod Biol Endocrinol 2009;7:126.

Mohammadi S, Jalali M, Nikravesh MR, Fazel A, Ebrahimzadeh A, Gholamin M, Sankian M. Effects of vitamin-E treatment on CatSper genes expression and sperm quality in the testis of the aging mouse. Iran J Reprod Med 2013; 1 I:989-998.

Mortimer ST, Swan MA, Mortimer D. Effect of seminal plasma on capacitation and hyperactivation in human spermatozoa. Hum Reprod 1998; 13:2139-21 46.

Muciaccia B, Padula F, Vicini E, Gandini L, Lenzi A, Stefanini M. Beta-chemokine receptors 5 and 3 are expressed on the head region of human spermatozoon. FASEB J 2005; 1 9:2048-2050.

Muciaccia B, Boitani C, Berloco BP, Nudo F, Spadetta G, Stefanini M, de Rooij DG, Vicini E. Novel stage classification of human spermatogenesis based on acrosome development. Biol Reprod 2013;89: I - 10.

Muratori M, Marchiani S, Tamburrino L, Tocci V, Failli P, Forti G, Baldi E. Nuclear staining identifies two populations of human sperm with different DNA fragmentation extent and relationship with semen parameters. Hum Reprod 2008;23: 1035- 1043.

Muratori M, Tamburrino L, Marchiani S, Cambi M, Olivito B, Azzari C, Forti G, Baldi E. Investigation on the origin of sperm DNA fragmentation: role of apoptosis, immaturity and oxidative stress. Mol Med 2015 [Epub ahead of print].

Nikpoor P, Mowla SJ, Movahedin M, Ziaee SA, Tiraihi T. CatSper gene expression in postnatal development of mouse testis and in subfertile men with deficient sperm motility. Hum Reprod 2004; 19: 124- 128.

Park EH, Kim do R, Kim HY, Park SK, Chang MS. Panax ginseng induces the expression of CatSper genes and sperm hyperactivation. Asian J Androl 20|4; | 6:845-85|.

Peedicayil J, Deendayal M, Sadasivan G, Shivaji S. Assessment of hyperactivation, acrosome reaction and motility characteristics of spermatozoa from semen of men of proven fertility and unexplained infertility. Andrologia 1997;29:209-218.

Publicover S, Barratt C. Reproductive biology: progesterone's gateway into sperm. Nature 201 I;47 I:3।3-3।4.

Qi H, Moran MM, Navarro B, Chong JA, Krapivinsky G, Krapivinsky L, Kirichok Y, Ramsey IS, Quill TA, Clapham DE. All four CatSper ion channel proteins are required for male fertility and sperm cell hyperactivated motility. Proc Natl Acad Sci USA 2007; 1 04: 12 1 19 - 1223.

Quill TA, Ren D, Clapham DE, Garbers DL. A voltage-gated ion channel expressed specifically in spermatozoa. Proc Natl Acad Sci USA 200I; 98: |2527- |253|.

Quill TA, Sugden SA, Rossi KL, Doolittle LK, Hammer RE, Garbers DL. Hyperactivated sperm motility driven by CatSper2 is required for fertilization. Proc Natl Acad Sci USA 2003; 100 : | 4869- 14874.

Ren D, Xia J. Calcium signaling through CatSper channels in mammalian fertilization. Physiology (Bethesda) 2010;25:165-175.

Ren D, Navarro B, Perez G, Jackson AC, Hsu S, Shi Q, Tilly JL, Clapham DE. A sperm ion channel required for sperm motility and male fertility. Nature 2001;413:603-609. 
Sagare-Patil V, Galvankar M, Satiya M, Bhandari B, Gupta SK, Modi D. Differential concentration and time dependent effects of progesterone on kinase activity, hyperactivation and acrosome reaction in human spermatozoa. Int J Androl 20I2;35:633-644.

Sagare-Patil V, Vernekar M, Galvankar M, Modi D. Progesterone utilizes the PI3K-AKT pathway in human spermatozoa to regulate motility and hyperactivation but not acrosome reaction. Mol Cell Endocrinol 2013; 374:82-91.

Sánchez-Cárdenas C, Servín-Vences MR, José O, Treviño CL, HernándezCruz A, Darszon A. Acrosome reaction and $\mathrm{Ca}^{2+}$ imaging in single human spermatozoa: new regulatory roles of $\left[\mathrm{Ca}^{2+}\right]_{\text {i. }}$. Biol Reprod 20I4; 91:67.

Smith JF, Syritsyna O, Fellous M, Serres C, Mannowetz N, Kirichok Y, Lishko PV. Disruption of the principal progesterone-activated sperma $\mathrm{Ca}^{2+}$ channel in a CatSper2-deficient infertile patient. Proc Natl Acad Sci USA 2013; 1 1 0:6823-6828.

Strünker T, Goodwin N, Brenker C, Kashikar ND, Weyand I, Seifert R, Kaupp UB. The CatSper channel mediates progesterone-induced $\mathrm{Ca}^{2+}$ influx in human sperm. Nature 201 I;47:382-386.

Suarez SS. Control of hyperactivation in sperm. Hum Reprod Update 2008; 14:647-657.

Sukcharoen N, Keith J, Irvine DS, Aitken RJ. Definition of the optimal criteria for identifying hyperactivated human spermatozoa at $25 \mathrm{~Hz}$ using in-vitro fertilization as a functional end-point. Hum Reprod 1995; 10:2928-2937.
Tamburrino L, Marchiani S, Minetti F, Forti G, Muratori M, Baldi E. The CatSper calcium channel in human sperm: relation with motility and involvement in progesterone-induced acrosome reaction. Hum Reprod 20। 4;29:4 I8-428.

Tavares RS, Mansell S, Barratt CL, Wilson SM, Publicover SJ, Ramalho-Santos J. $p, p^{\prime}$-DDE activates CatSper and compromises human sperm function at environmentally relevant concentrations. Hum Reprod 2013;28:3167-3177.

Tesarik J, Mendoza C. Defective function of a nongenomic progesterone receptor as a sole sperm anomaly in infertile patients. Fertil Steril 1992 58:793-797.

Visser L, Westerveld GH, Xie F, van Daalen SK, van derVeen F, Lombardi MP, Repping S. A comprehensive gene mutation screen in men with asthenozoospermia. Fertil Steril 20 I ; 95: 1020-1024.el -9.

Wang C, Lee GS, Leung A, Surrey ES, Chan SY. Human sperm hyperactivation and acrosome reaction and their relationships to human in vitro fertilization. Fertil Steril 1993;59: 1221 - 1227.

Wang Y, Shi J, Li L, Liu D, Li L, Tang C, Li J. Adverse effects of $2,2^{\prime}, 4,4^{\prime}-$ tetrabromodiphenyl ether on semen quality and spermatogenesis in male mice. Bull Environ Contam Toxicol 2013;90:5I-54.

World Health Organization. WHO Laboratory Manual for the Examination and Processing of Human Semen, 5th edn. Cambridge, UK: Cambridge University Press, 2010.

Xia J, Reigada D, Mitchell CH, Ren D. CATSPER channel-mediated $\mathrm{Ca}^{2+}$ entry into mouse sperm triggers a tail-to-head propagation. Biol Reprod 2007;77:55I - 559 . 